\title{
INVERSE ELEMENTARY DIVISOR PROBLEM FOR NONNEGATIVE MATRICES ${ }^{1}$
}

\author{
HENRYK MINC
}

\begin{abstract}
Given a diagonalizable positive matrix $A$, there exists a positive matrix with the same spectrum as $A$, and with arbitrarily prescribed elementary divisors, provided that elementary divisors corresponding to nonreal eigenvalues occur in conjugate pairs. It is also shown that a similar result holds for doubly stochastic matrices.
\end{abstract}

1. Introduction. One of the most important unsolved problems of linear algebra is the inverse eigenvalue problem for nonnegative matrices: to determine necessary and sufficient conditions that a given $n$-tuple of complex numbers be the spectrum of an $n \times n$ nonnegative matrix, i.e., matrix with nonnegative entries. (For information on the status of the problem and bibliography see [1, 2].) The inverse elementary divisor problem for nonnegative matrices asks for necessary and sufficient conditions for a given matrix to be similar to a nonnegative matrix, or equivalently, necessary and sufficient conditions for the existence of a nonnegative matrix with prescribed elementary divisors. The inverse elementary divisor problem clearly contains the inverse eigenvalue problem, and it is also unsolved. In this paper we consider the inverse elementary divisor problem modulo the inverse eigenvalue problem: given a nonnegative matrix $A$, does there exist a nonnegative matrix with the same spectrum as $A$ and with arbitrarily prescribed elementary divisors (provided that prescribed elementary divisors corresponding to nonreal eigenvalues occur in conjugate pairs)? We show (Theorem 1) that the answer is in the affirmative in case $A$ is a diagonalizable positive matrix. It is not known if the theorem holds for a general nonnegative matrix $A$.

In Theorem 2 we establish a similar result for doubly stochastic matrices. It should be noted that in the case of doubly stochastic matrices the condition that $A$ be positive cannot be relaxed (see §2).

2. Results. Let $J_{n}$ denote the $n \times n$ matrix all of whose entries are $1 / n$, and let $E_{i j}$ be the $n \times n$ matrix with 1 in the $(i, j)$ position and 0 's elsewhere. Denote the $j$ th column of $S$ by $S^{(j)}$, and the submatrix obtained from $S$ by deleting rows $i_{1}, i_{2}, \ldots, i_{k}$ and columns $j_{1}, j_{2}, \ldots, j_{k}$, by $S\left(i_{1}, i_{2}, \ldots, i_{k} \mid j_{1}, j_{2}, \ldots, j_{k}\right)$. If $S$ is a nonsingular matrix we denote the $(i, j)$ entry of $S^{-1}$ by $\hat{s}_{i j}$.

Let $A=\left(a_{i j}\right)$ be an $n \times n$ diagonalizable positive matrix with eigenvalues $\lambda_{1}, \lambda_{2}, \ldots, \lambda_{n}$ which are ordered as follows: $\lambda_{1}>\lambda_{2} \geqslant \cdots \geqslant \lambda_{p}$ are real, and the

Received by the editors November 12, 1980 and, in revised form, April 17, 1981.

1980 Mathematics Subject Classification. Primary 15A18, 15A48, 15A51.

${ }^{1}$ Research supported by the Air Force Office of Scientific Research under Grant AFOSR-79-0127A. 
other $n-p$ eigenvalues are complex nonreal; equal eigenvalues are consecutive, and $\lambda_{t}$ is conjugate to $\lambda_{q+t}$ for $t=p+1, p+2, \ldots, p+q$, where $q=(n-p) / 2$.

THEOREM 1. Given a diagonalizable positive matrix $A$, there exists a positive matrix with the same spectrum as $A$, and with arbitrarily prescribed elementary divisors, provided that elementary divisors corresponding to nonreal eigenvalues occur in conjugate pairs.

Our second result is an analogue of Theorem 1 for doubly stochastic matrices, and extends the result in [3, Theorem 4] to matrices with complex eigenvalues. $A$ complex matrix is called doubly quasi-stochastic if all its row sums and column sums are 1. A necessary and sufficient condition that an $n \times n$ matrix $A$ be quasi-stochastic is that

$$
A J_{n}=J_{n} A=J_{n} \text {. }
$$

A nonnegative (real) doubly quasi-stochastic matrix is called doubly-stochastic.

The existence of a nonnegative matrix with a prescribed spectrum does not imply the existence of a doubly stochastic matrix with the same spectrum, even if the maximal eigenvalue is 1 . For example, the eigenvalues of a nonnegative matrix of the form

$$
\left[\begin{array}{lll}
0 & 1 & 0 \\
1 & 0 & 0 \\
0 & 0 & t
\end{array}\right]
$$

are $1,-1, t$, for any $1>t \geqslant 0$, but there exists no $3 \times 3$ doubly stochastic matrix with these eigenvalues. The situation with respect to the inverse elementary divisor problems is similar. It was shown in [1, Theorem 3(b)] that there exists no doubly stochastic $3 \times 3$ matrix with elementary divisors $\lambda-1$ and $\left(\lambda+\frac{1}{2}\right)^{2}$, although the doubly stochastic matrix $\frac{3}{2} J_{3}-\frac{1}{2} I_{3}$ has eigenvalues $1,-\frac{1}{2},-\frac{1}{2}$, and the rowstochastic matrix

$$
\frac{1}{2}\left[\begin{array}{lll}
0 & 2 & 0 \\
1 & 0 & 1 \\
1 & 1 & 0
\end{array}\right]
$$

has elementary divisors $\lambda-1$ and $\left(\lambda+\frac{1}{2}\right)^{2}$. If, however, the prescribed eleme tary divisors involve eigenvalues of a diagonalizable positive matrix, then the case of doubly stochastic matrices is analogous to that of general nonnegative matrices.

THEOREM 2. Given a diagonalizable positive doubly stochastic matrix $A$, there exists a positive doubly stochastic matrix with the same spectrum as $A$, and with arbitrarily prescribed elementary divisors, provided that elementary divisors corresponding to nonreal eigenvalues occur in conjugate pairs.

3. Proofs. We start with a simple lemma.

LEMMA. Let $S$ be an $n \times n$ nonsingular matrix whose first $p$ columns are real and, for $t=p+1, p+2, \ldots, p+q, q=(n-p) / 2$, columns $t$ and $q+t$ are conjugate. Then rows $t$ and $q+t$ of $S^{-1}$ are conjugate for $t=p+1, p+2, \ldots, p+q$. 
Proof. Transpose columns $t$ and $q+t$ of $S, t=p+1, p+2, \ldots, p+q$; the resulting matrix is $\bar{S}$, the conjugate of $S$. Hence

$$
\operatorname{det}(S)=(-1)^{q} \overline{\operatorname{det}(S)} \text {. }
$$

Thus $\operatorname{det}(S)$ is real if $q$ is even, and pure imaginary if $q$ is odd. Similarly,

$$
\operatorname{det}(S(i, j \mid t, q+t))
$$

is real or pure imaginary according as $q$ is odd or even, for any $i, j$ and $t$, $1 \leqslant i<j \leqslant n, p<t \leqslant p+q$. It follows that

$$
\operatorname{det}(S(i, j \mid t, q+t)) / \operatorname{det}(S)
$$

is pure imaginary.

We have

$$
\hat{s}_{t j}=(-1)^{t+j} \operatorname{det}(S(j \mid t)) / \operatorname{det}(S),
$$

and expanding $\operatorname{det}(S(j \mid t))$ by column $q+t$, we obtain

$$
\hat{s}_{t j}=(-1)^{t+j} \sum_{\substack{i=1 \\ i \neq j}}^{n}(-1)^{i^{\prime}+q+t-1} s_{i, q+t} \operatorname{det}(S(i, j \mid t, q+t)) / \operatorname{det}(S),
$$

where

$$
i^{\prime}= \begin{cases}i & \text { if } i<j \\ i-1 & \text { if } i>j\end{cases}
$$

Now,

$$
\overline{s_{i, q+t}}=s_{i, t}
$$

and

$$
\overline{\operatorname{det}(S(i, j \mid t, q+t)) / \operatorname{det}(S)}=-\operatorname{det}(S(i, j \mid t, q+t)) / \operatorname{det}(S) .
$$

Therefore for $t=p+1, p+2, \ldots, p+q$, and $j=1,2, \ldots, n$,

$$
\begin{aligned}
\overline{\hat{s}_{t j}} & =(-1)^{q+t+j} \sum_{\substack{i=1 \\
i \neq j}}^{n}(-1)^{i^{\prime}+t} s_{i t} \operatorname{det}(S(i, j \mid t, q+t)) / \operatorname{det}(S) \\
& =(-1)^{q+t+j} \operatorname{det}(S(j \mid q+t)) / \operatorname{det}(S)=\hat{s}_{q+t, j} .
\end{aligned}
$$

Proof of Theorem 1. Let $D=\operatorname{diag}\left(\lambda_{1}, \lambda_{2}, \ldots, \lambda_{n}\right)$, where $\lambda_{1}, \lambda_{2}, \ldots, \lambda_{n}$ are the eigenvalues of $A$, ordered as in $\S 2$. Let $S$ be a nonsingular matrix whose $j$ th column is an eigenvector corresponding to $\lambda_{j}, j=1,2, \ldots, n$, and whose columns $j$ and $p+j$ are conjugate, $j=p+1, p+2, \ldots, p+q$, where $q=(n-p) / 2$. Then

$$
S^{-1} A S=D \text {. }
$$

Now, let $X$ be the subset of $\{2,3, \ldots, n-1\}$ so that $D+\Sigma_{t \in X} E_{t, t+1}$ is the Jordan normal form of the required matrix. We show that the matrix

$$
B=\left(b_{i j}\right)=S \sum_{t \in X} E_{t, t+1} S^{-1}
$$


is real. Note that the first $p$ rows of $S^{-1}$ are real. For, they must be multiples of real vectors (being eigenvectors of $A^{T}$ corresponding to real eigenvalues), and $S^{-1} S=$ $I_{n}$. Further, by the lemma, rows $t$ and $q+t$ of $S^{-1}$ are conjugate for $t=p+1$, $p+2, \ldots, p+q$. Now,

$$
b_{i j}=\sum_{t \in X} s_{i t} \hat{s}_{t+1, j}
$$

$i, j=1, \ldots, n$. For $t=1, \ldots, p-1$, both $s_{i t}$ and $\hat{s}_{t+1, j}$ are real. For any $t$, $p \leqslant t<p+q$, if $t$ belongs to $X$ (note that $p, q, n \notin X$ ), then $t+q \in X$, and

$$
\overline{s_{i t}}=s_{i, t+q} \text { and } \overline{\hat{s}_{t+1, j}}=\hat{s}_{t+q+1, j}
$$

for all $i$ and $j$. Therefore

$$
\begin{aligned}
\sum_{\substack{t=p \\
t \in X}}^{n} s_{i t} \hat{s}_{t+1, j} & =\sum_{\substack{t=p+1 \\
t \in X}}^{p+q-1}\left(s_{i t} \hat{s}_{t+1, j}+s_{i, t+q} \hat{s}_{t+q+1, j}\right) \\
& =2 \sum_{\substack{t=p+1 \\
t \in X}}^{p+q-1} \operatorname{Re}\left(s_{i t} \hat{s}_{t+1, j}\right) .
\end{aligned}
$$

Hence $b_{i j}$ is real for all $i$ and $j$.

Now, $S D S^{-1}=A$ is positive, and therefore $A+\varepsilon B$ is positive for sufficiently small positive $\varepsilon$. Thus

$$
A+\varepsilon B=S\left(D+\varepsilon \sum_{t \in X} E_{t, t+1}\right) S^{-1}
$$

is positive and has the required elementary divisors.

Proof of TheOrem 2. As in the proof of Theorem 1 , let $D=$ $\operatorname{diag}\left(\lambda_{1}, \lambda_{2}, \ldots, \lambda_{n}\right)$, where $\lambda_{1}=1, \lambda_{2}, \ldots, \lambda_{n}$ are the eigenvalues of $A$ ordered as in $\$ 2$, and let $D+\sum_{t \in X} E_{t, t+1}$ be the Jordan normal form of the required matrix. We construct a nonsingular matrix $S$ as follows. The $j$ th column of $S$ is an eigenvector corresponding to $\lambda_{j}, j=1,2, \ldots, n$, the first column being $\left[\begin{array}{llll}1 & 1 & \cdots\end{array}\right]^{T} / \sqrt{n}$, columns $2,3, \ldots, p$ being real and columns $j$ and $j+p$ being conjugate for $j=p+1, p+2, \ldots, q$. Then, as in the proof of Theorem 1 , the matrix

$$
C=S\left(D+\varepsilon \sum_{t \in X} E_{t, t+1}\right) S^{-1}
$$

is positive for sufficiently small positive $\varepsilon$. It remains to show that $C$ is doubly quasi-stochastic and thus stochastic.

The first row of $S^{-1}$ is $\left[\begin{array}{lll}1 & 1 & \cdots\end{array}\right] / \sqrt{n}$, since it is an eigenvector corresponding to 1 of positive doubly stochastic matrix $A^{T}$. It follows that all the other row sums of $S^{-1}$ and all the column sums of $S$, except the first, are zero. Therefore $S^{-1} J_{n}$ is the matrix whose first row is $\left[\begin{array}{llll}1 & 1 & \cdots & \cdots\end{array}\right] / \sqrt{n}$ and the other $n-1$ rows are zero, and $J_{n} S$ is the transpose of $S^{-1} J_{n}$. If we write $D+\varepsilon \sum_{t \in X} E_{i, i+1}$ in the form $1+G$, where $G$ is $(n-1) \times(n-1)$, then

$$
(1+G)\left(S^{-1} J_{n}\right)=S^{-1} J_{n},
$$


and

$$
J_{n} S(1+G)=J_{n} S .
$$

It follows that

$$
C J_{n}=S\left((1+G) S^{-1} J_{n}\right)=S\left(S^{-1} J_{n}\right)=J_{n},
$$

and similarly

$$
J_{n} C=\left(J_{n} S(1+G)\right) S^{-1}=\left(J_{n} S\right) S^{-1}=J_{n} .
$$

\section{REFERENCES}

1. Abraham Berman and Robert J. Plemmons, Nonnegative matrices in the mathematical sciences, Academic Press, New York, 1979.

2. F. R. Gantmacher, The theory of matrices, Vol. 2, Chelsea, New York, 1959.

3. Henryk Minc, Inverse elementary divisor problem for doubly stochastic matrices, Linear and Multilinear Algebra (to appear).

INSTITUTE FOR ALGEBRA AND COMBINATORICS AND DEPARTMENT OF MATHEMATICS, UNIVERSITY OF California, Santa Barbara, California 93106 